\title{
Aharonov-Bohm effect as a probe of interaction between magnetic impurities
}

\author{
Victor M. Galitski, ${ }^{1}$ Maxim G. Vavilov, ${ }^{2}$ and Leonid I. Glazman ${ }^{3}$ \\ ${ }^{1}$ Condensed Matter Theory Center, Department of Physics, University of Maryland, College Park, MD 20742 \\ ${ }^{2}$ Center for Materials Sciences and Engineering, Massachusetts Institute of Technology, Cambridge, MA 02139 \\ ${ }^{3}$ Theoretical Physics Institute, School of Physics and Astronomy, University of Minnesota, Minneapolis, MN 55455
}

\begin{abstract}
We study the effects of the RKKY interaction between magnetic impurities on the mesoscopic conductance fluctuations of a metal ring with dilute magnetic impurities. At sufficiently low temperatures and strong magnetic fields, the loss of electron coherence occurs mainly due to the scattering off rare pairs of strongly coupled magnetic impurities. We establish a relation between the dephasing rate and the distribution function of the exchange interaction within such pairs. In the case of the RKKY exchange interaction, this rate exhibits $1 / B^{2}$ behavior in strong magnetic fields. We demonstrate that the Aharonov-Bohm conductance oscillations may be used as a probe of the distribution function of the exchange interaction between magnetic impurities in metals.
\end{abstract}

PACS numbers: 03.65.Yz, 72.15.Rn

The exchange interaction between magnetic impurities and itinerant electrons drastically affects electron transport in metals. Recent experiments [1, 2, 3, 4] have revealed a significant effect of magnetic impurities on the coherent transport (weak localization and conductance fluctuations) and electron energy relaxation in metals with even a tiny concentration of magnetic impurities. In measurements [1], the amplitude of the " $h / e$ " Aharonov-Bohm (AB) oscillations in mesoscopic metal rings showed a strong dependence on the value of the applied field $B$, with the oscillations being suppressed at low fields and restored at relatively high fields. This observation is consistent with the picture of uncorrelated magnetic impurities frozen by a high magnetic field [5, 6].

The exchange coupling between magnetic impurities and electrons also gives rise to the Ruderman-Kittel-KasuyaYosida (RKKY) interaction between spins of magnetic impurities [7]. At sufficiently low temperatures the RKKY interaction between magnetic impurities affects the electron kinetics in metals [8]. The observation of the effect of interaction between magnetic impurities was pursued in experiments [4], where the temperature dependence of the resistivity and the electron phase relaxation rate in diluted magnetic alloys were investigated.

In this paper, we study the effect of the RKKY interaction on the $\mathrm{AB}$ oscillations of the conductance [9, 10] of mesoscopic metal rings containing magnetic impurities. We show that the amplitude of the $\mathrm{AB}$ oscillations may be used as an experimental tool to probe the statistics of the exchange interaction between magnetic impurities. Pairs of magnetic impurities coupled by the exchange interaction $V$ comparable to the Zeeman energy $g \mu B$ of an impurity in a magnetic field $B$, play a special role (here $g$ is the $g$-factor of a magnetic impurity and $\mu$ is the electron Bohr's magneton). The dynamics of such resonant pairs is not quenched by the field $B$, while the other spins are frozen. The resonant pairs have the strongest effect on the amplitude of the $\mathrm{AB}$ conductance oscillations. The oscillations are characterized by the following correlation function [10],

$$
\left\langle G_{\Phi}(B) G_{\Phi+\Delta \Phi}(B)\right\rangle=\frac{\alpha e^{4}}{\pi^{2} \hbar^{2}} \sum_{k=0}^{\infty} \mathcal{A}_{k}(B) \cos \left(2 \pi k \frac{\Delta \Phi}{\Phi_{0}}\right),
$$

where $G_{\Phi}(B)$ is the conductance of the ring in the presence of a magnetic flux $\Phi, \Phi_{0}=h c / e$ is the flux quantum, and $\alpha$ is a geometry-dependent numerical factor. The strength of the $\mathrm{AB}$ effect is determined by the amplitudes $\mathcal{A}_{k}(B)$ with $k \neq 0$. In the limit of low temperatures and high magnetic fields, we find

$$
\mathcal{A}_{k}(B) \propto \exp \left(-\frac{k L}{L_{\varphi}}\right), \quad \frac{1}{L_{\varphi}^{2}} \propto T P(g \mu B) .
$$

Here $L$ is the ring circumference, $T$ is the electron temperature and $P(g \mu B)$ is the density of magnetic impurity pairs coupled by exchange interaction of strength $V=g \mu B$. Thus, the measurement of the $\mathrm{AB}$ amplitudes $\mathcal{A}_{k}(B)$ allows one to "scan" the distribution function $P(V)$ of the interaction strength between magnetic impurities. The applicability of Eq. (2) requires $T \ll \bar{V} \ll g \mu B$, where $\bar{V}$ is the typical strength of the interaction between magnetic impurities.

The $\mathrm{AB}$ conductance oscillations, being an interference phenomenon [9, 10], are very sensitive to any changes in disorder realization [11, 12]. Particularly, temporal changes in the disorder configuration during the measurement process suppress the amplitudes $\mathcal{A}_{k}$ of the conductance correlation function (11). This suppression is a result of the conductance self-averaging during the measurement. A possible mechanism for such suppression comes from the evolution of spin configuration in the system of magnetic impurities [5] on the time scale defined by impurity spin relaxation. This scale is much shorter than the conductance measurement time. However, if the spins of magnetic impurities are quenched, they do not suppress the amplitude of the conductance fluctuations.

A way to quench the spin dynamics of magnetic impurities is to apply a magnetic field. In the system of non-interacting magnetic impurities, the magnetic field $B$ yields an exponential suppression of the spin-flip scattering rate $\gamma_{\mathrm{s}}$ : if the Zeeman energy splitting exceeds temperature, $g \mu B \gg T$, then 
$\gamma_{\mathrm{s}} \propto \exp [-g \mu B / T]$, see [5, 6]. Interaction between magnetic impurities may weaken the spin quenching by magnetic field, if the interaction strength between impurities is comparable with the Zeeman splitting. We consider strong magnetic fields when the majority of spins are frozen by the applied field. Due to random positions of magnetic impurities, there exist strongly interacting pairs for which the dynamics is not quenched. Such pairs effectively act as two-level systems, and provide the main contribution to the suppression of the $\mathrm{AB}$ conductance oscillations. We note that in strong magnetic fields (the Zeeman splitting exceeds the typical strength of the interaction between magnetic impurities) the probability to find three or more strongly coupled magnetic impurities is much smaller than the probability of finding a strongly coupled pair. In evaluating $\gamma_{\mathrm{s}}$, we account only pairs of magnetic impurities, following Refs. [13, 14].

The competition between the antiferromagnetic exchange $V>0$ and the Zeeman splitting results in the degeneracy of spin states of impurity pairs for which the condition $V=g \mu B$ is satisfied. The spin dynamics of such pairs is not quenched, and they contribute to the electron dephasing. The remaining impurity spins are frozen by the applied magnetic field or interactions and do not suppress the conductance fluctuations (we note that the dynamics of ferromagnetically coupled spins, $V<0$, is quenched even stronger than the dynamics of non-interacting spins). Therefore, the amplitudes $\mathcal{A}_{k}(B)$, see Eq. (1), are determined by the magnetic impurity pairs characterized by the exchange interaction strength $V$ of the order of the Zeeman splitting: $|V-g \mu B| \lesssim T$.

We focus on the limit of strong spin-orbit scattering (the spin-orbit scattering rate is supposed to be much larger than the scattering rate off magnetic impurities). The interaction of an electron with a magnetic impurity is described by the exchange Hamiltonian $\mathcal{H}_{\mathrm{ex}}=\mathcal{J} \hat{\boldsymbol{S}} \hat{\boldsymbol{\sigma}}$, where $\mathcal{J}$ is the exchange coupling constant, $\hat{S}$ is the spin of a magnetic impurity, and $\hat{\sigma}$ is the electron spin density. The exchange coupling leads to the effective RKKY interaction between magnetic impurities. If the distance between the impurities in a pair is smaller than the spin-orbit scattering length $L_{\mathrm{so}}$, the coupling between the impurity spins $\hat{S}_{1,2}$ is isotropic [15] and the corresponding Hamiltonian is

$$
\mathcal{H}_{\mathrm{S}}^{(2)}=V \hat{\boldsymbol{S}}_{1} \hat{\boldsymbol{S}}_{2}+g \mu B\left[\hat{S}_{1 z}+\hat{\boldsymbol{S}}_{2 z}\right] .
$$

Here $V$ is the RKKY coupling. The eigenstates of this Hamiltonian $|\xi\rangle=|J, M\rangle$ are classified by the total spin $J$ and the projection $M$ of the total spin on the direction of the magnetic field. The energy spectrum of Eq. (3) has the form

$$
E_{J, M}=\frac{V}{2}[J(J+1)-S(S+1)]+g \mu B M .
$$

Below we consider spin-1/2 magnetic impurities, in which case there are four energy levels corresponding to three $J=1$ states and one $J=0$ state.

We calculate the spin-flip scattering rate off pairs of mag- netic impurities

$$
\gamma_{\mathrm{s}}(\varepsilon)=\frac{4}{3 \tau_{\mathrm{s}}} \int d V P(V)\left[K(\varepsilon, V)-\left\langle S_{z}\right\rangle^{2}\right],
$$

where $\tau_{\mathrm{s}}^{-1}=(3 \pi / 2) n_{s} v \mathcal{J}^{2}$ is the scattering rate off an isolated impurity at zero magnetic field, $v$ is the electron density of states at the Fermi level and $n_{\mathrm{s}}$ is the concentration of magnetic impurities. The term $4 K(\varepsilon, V) / 3 \tau_{\mathrm{s}}$ in Eq. (5) represents the scattering rate of an electron with energy $\varepsilon$ off a magnetic impurity, which belongs to a pair characterized by the exchange interaction strength $V$. This rate can be obtained from the Fermi golden rule:

$$
K(\varepsilon, V)=\sum_{\xi \xi^{\prime}} \frac{e^{-E_{\xi} / T}}{Z}\left|\left\langle\xi|\hat{\boldsymbol{S}}| \xi^{\prime}\right\rangle\right|^{2} \frac{1+e^{\varepsilon / T}}{1+e^{\left(\varepsilon-E_{\xi}+E_{\xi^{\prime}}\right) / T}},
$$

where $Z=\sum_{\xi} e^{-E_{\xi} / T}$ is the partition function. The polarization $\left\langle S_{z}\right\rangle=\sum_{\xi} e^{-E_{\xi} / T}\left\langle\xi\left|\hat{S}_{z}\right| \xi\right\rangle / Z$ of magnetic impurities reduces fluctuations of impurity spins and also reduces $\gamma_{\mathrm{s}}$; this effect is described by the $4\left\langle S_{z}\right\rangle^{2} / 3 \tau_{\mathrm{s}}$ term in Eq. (6). The rate $\gamma_{\mathrm{s}}(\varepsilon)$ represents the result of averaging over the strength of the exchange coupling $V$ with the weight $P(V)$. Here $P(V)$ denotes the distribution function of the exchange coupling $V$ for pairs of magnetic impurities, which we discuss later.

We consider Zeeman splitting $g \mu B$ exceeding both temperature $T$ and the typical inter-impurity coupling $\bar{V}$. In this case, the strength of interaction between magnetic impurities becomes comparable with $g \mu B$ at distances much shorter than the typical distance between magnetic impurities $n_{\mathrm{s}}^{-1 / 3}$, which justifies the use of the virial expansion method. If the characteristic scale for variation of $P(V)$ is larger than temperature $T$, we obtain from (5) and (6)

$$
\gamma_{\mathrm{s}}(\varepsilon)=\frac{T P(g \mu B)}{3 \tau_{\mathrm{s}}}\left(1+\frac{2 \varepsilon}{T} \operatorname{coth} \frac{\varepsilon}{2 T}\right), \quad|\varepsilon| \ll g \mu B .
$$

The spin flip rate (7) takes into account the scattering processes which change the state of impurity spins in a pair from $J=1$ and $M=-1$ to $J=0$ and vice versa. Therefore, a pair of impurity spins coupled with the strength $V-g \mu B \lesssim T$ acts as a two level system [16].

We emphasize that, in general, the scattering rate off magnetic impurities is not a single universal parameter, which determines all transport properties of an electron liquid with embedded magnetic impurities. Various properties of the conductance, such as the weak localization correction [17] and the conductance fluctuations, are determined by different parameters characterizing scattering off magnetic impurities (see Ref. [6] for a detailed analysis). Here Eq. (7) defines the dephasing rate $\gamma_{\mathrm{s}}(\varepsilon)$, which limits the amplitude of the Aharonov-Bohm oscillations, Eq. (1).

The amplitudes $\mathcal{A}_{k}$ in Eq. (1) in the limit of weak scattering off magnetic impurities, $\gamma_{\mathrm{s}}(T) \ll T, D / L_{\mathrm{so}}^{2}$, can be written in the form [6]

$$
\mathcal{A}_{k}=\frac{D^{3 / 2}}{T^{2} R^{3}} \int \frac{e^{-k L} \sqrt{\gamma_{\mathrm{s}}(\varepsilon) / D}}{\sqrt{\gamma_{\mathrm{s}}(\varepsilon)}} \frac{d \varepsilon}{\cosh ^{4}(\varepsilon / 2 T)},
$$


where $D$ is the diffusion coefficient. Substituting Eq. (7) into Eq. (8), we find the amplitudes of the conductance correlation function harmonics. If the circumference of the ring $L$ is much larger than the coherence length $L_{\varphi}$, then the saddle point approximation is applicable and we find

$$
\begin{aligned}
\mathcal{A}_{k} & =\sqrt{\frac{15}{k}} \frac{L_{T}^{2} L_{\varphi}^{3 / 2}}{[L / 2 \pi]^{7 / 2}} \exp \left(-k \frac{L}{L_{\varphi}}\right), \\
\frac{1}{L_{\varphi}^{2}} & =\frac{\gamma_{\mathrm{s}}}{D}=\frac{5 T}{3 \tau_{\mathrm{s}} D} P(g \mu B),
\end{aligned}
$$

cf. Eq. (2). Here $L_{T}=\sqrt{D / T}$ is the thermal length, $\gamma_{\mathrm{s}}=\gamma_{\mathrm{s}}(\varepsilon=0)$, and the coherence length $L_{\varphi}$ in a magnetic field $B$ is determined by the probability $P(V)$ of finding a pair of two magnetic impurities antiferromegnetically interacting with each other with the strength $V=|g \mu B|$, see Eq. (7). Therefore, the amplitude of the conductance fluctuations can be used to probe the distribution function $P(V)$. Below we discuss the appropriate distribution functions for the RKKY interaction in the limits of strong and weak disorder.

The strength of the RKKY coupling is determined by the electron wave functions at the positions of magnetic impurities. In a disordered metal, the values of the wave functions at distances $r$ larger than the mean free path $l$ are nearly uncorrelated, and the average value of RKKY coupling over disorder is exponentially small: $\langle V(r)\rangle \sim \exp (-r / l)$. However, the values of $V(r)$ in a given configuration of disorder remain $\propto 1 / r^{3}$ even in the "strong disorder" limit $r \gg l$, as one can see [15, 18, 19] from the variance of RKKY coupling, $\left\langle V^{2}(r)\right\rangle=(3 / 4) C^{2} / r^{6}$ with $C=v \mathcal{T}^{2} / 2 \pi$.

We emphasize that in a disordered metal the interaction strength between two magnetic impurities is not uniquely determined by the distance between these impurities, but also depends on disorder realization. The full distribution function $p(V, r)$ of $V(r)$ is not known even for a metal with $k_{F} l \gg 1$. However, from the $1 / r^{6}$ behavior of the variance on the distance between magnetic impurities, we assume that the distribution function $p(V, r)$ has the following scaling form $p(V, r)=r^{3} \zeta\left(r^{3} V / C\right) / C$. For magnetic impurities randomly positioned in space according to the Poisson distribution, the probability of finding the nearest neighbor at a distance $r \ll n_{\mathrm{s}}^{-1 / 3}$ is equal to $n_{\mathrm{s}}$. To derive the probability distribution function of the RKKY interaction strength, we average over the distance between magnetic impurities $r$ :

$$
P_{\mathrm{dis}}(V)=\int 4 \pi r^{2} \frac{r^{3} \zeta\left(r^{3} V / C\right)}{C} d r=\frac{\bar{V}}{V^{2}}, \bar{V}=\frac{4}{3} \eta \pi C n_{\mathrm{s}} .
$$

Here we introduced the typical interaction strength $\bar{V}$ between magnetic impurities at the distance $n_{\mathrm{s}}^{-1 / 3}$ and $\eta=\int_{0}^{\infty} x \zeta(x) d x$ is a numerical factor. We emphasize that Eq. (10) is applicable for impurities at $r \ll n_{\mathrm{s}}^{-1 / 3}$, i. e., for $V \gg \bar{V}$, where the virial approximation is justified. According to Eq. (7), the corresponding scattering rate is

$$
\gamma_{\mathrm{s}}=\frac{5}{3 \tau_{\mathrm{s}}} \frac{T \bar{V}}{(g \mu B)^{2}},
$$

in sharp contrast to the exponential decay rate for isolated magnetic impurities in a strong magnetic field $B[5,6]$.

We note here that the scattering rate off non-interacting magnetic impurities calculated to fourth order in the exchange coupling $\mathcal{J}$ also has a power law dependence on the applied magnetic field [6]: $\gamma_{\mathrm{s}}^{(4)} \propto T^{2} /(g \mu B)^{2}$. However, this rate becomes smaller than the rate $\gamma_{\mathrm{s}}$, Eq. 11], at $T \lesssim \bar{V}$. Therefore, in the low-temperature and strong magnetic field limit, $T \ll \bar{V} \ll g \mu B$, the amplitudes $\mathcal{A}_{k}(B)$ of the " $h / e$ " AharonovBohm oscillations, Eq. (2), are determined by electron scattering off magnetic impurities coupled in pairs. The corresponding coherence length $L_{\varphi}$ and dephasing rate $\gamma_{\mathrm{s}}$ are given by Eqs. 9b) and 111, respectively.

As the magnetic field increases, suppression of the $A B$ amplitudes is determined by impurity pairs with the typical separation of order $r(B)=[C /(g \mu B)]^{1 / 3}$, where the factor $C=\nu \mathcal{J}^{2} / 2 \pi$ is determined by the renormalized exchange constant $\mathcal{J}$ due to the Kondo effect and can be estimated as

$$
C(r)=\frac{2}{\pi v} \ln ^{-2}\left[\frac{\max \left\{v_{\mathrm{F}} / r, g \mu B, T\right\}}{T_{\mathrm{K}}}\right] .
$$

A question arises: what happens when the distance becomes smaller than the elastic mean free path $l$ ? At such distances, the mesoscopic fluctuations of the RKKY exchange interaction are negligible, therefore

$$
V(r)=\frac{C}{r^{3}} \cos 2 p_{\mathrm{F}} r .
$$

The corresponding distribution function $P(V)=P_{\mathrm{c}}(V)$,

$$
P_{\mathrm{c}}(V)=\int d^{3} \mathbf{r} \delta\left[V-\frac{C}{r^{3}} \cos \left(2 p_{\mathrm{F}} r\right)\right] .
$$

is non-monotonic and possesses cusps $1 / \sqrt{V_{n}-V}$ at the maxima of the RKKY interaction $V_{n}=$ $\left(8 / \pi^{2}\right)\left(E_{\mathrm{F}} / n^{3}\right) \ln ^{-2}\left[E_{\mathrm{F}} / n T_{\mathrm{K}}\right]$; here the maxima are labeled with the index $n \sim\left(E_{\mathrm{F}} / g \mu B\right)^{1 / 3} \gg 1$ and $E_{\mathrm{F}}$ is the Fermi energy. These singularities should reveal themselves in high-field measurements [as defined below in Eq. [15] of the conductance correlation function, being however partially smeared out by temperature $T$ and non-magnetic disorder.

The oscillations of the amplitude of the conductance correlation function due to the singularities of $P(V)$ can be resolved if the distance between the neighboring maxima $\Delta V_{n}=$ $V_{n}-V_{n+1}$ is larger than the temperature; this condition is satisfied at $3 V_{n} / n \gg T$. The singularities in $P(V)$ are not smeared by disorder if the magnetic impurities in a pair are separated by distances smaller than the mean free path. The latter condition yields $n \ll l p_{\mathrm{F}} / \pi$. Keeping in mind that the distance between magnetic impurities being probed is to be much larger than the lattice spacing, we obtain the following set of conditions for the observability of the modulation of the amplitudes $\mathcal{A}_{k}(B)$ of the $\mathrm{AB}$ conductance oscillations:

$$
\max \left\{\left(\frac{T}{E_{\mathrm{F}}}\right)^{3 / 4}, \frac{1}{\left(p_{\mathrm{F}} l\right)^{3}}\right\} \ll \frac{g \mu B}{E_{\mathrm{F}}} \ll \ln ^{-2}\left[\frac{E_{\mathrm{F}}}{T_{\mathrm{K}}}\right] .
$$




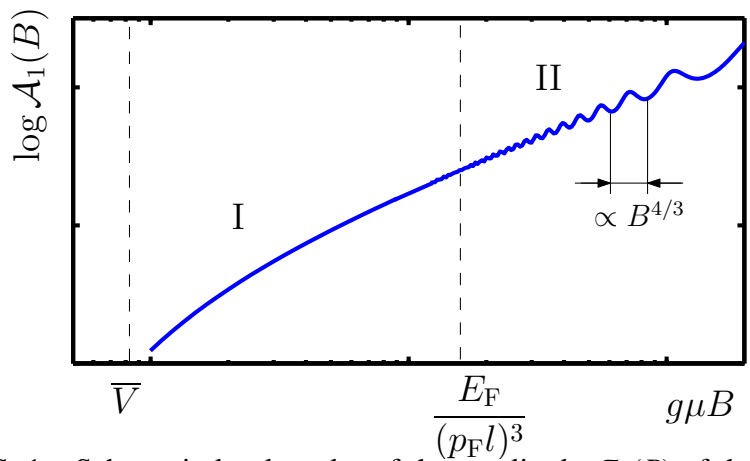

FIG. 1: Schematic log-log plot of the amplitude $\mathcal{A}_{1}(B)$ of the AB oscillations as a function of the Zeeman splitting $g \mu B$. Two regimes are shown: I) interacting pairs of magnetic impurities separated by distances larger than the mean free path $l$ described by Eqs. 9a, (9b), and (11); II) interacting pairs of magnetic impurities separated by distance smaller than the mean free path $l$.

Estimating $E_{\mathrm{F}} \sim 10^{4} \mathrm{~K}$ and $p_{\mathrm{F}} l \sim 100$, we conclude that the magnetic field can be as low as $g \mu B \sim 1 \mathrm{~K}$ and the temperature as high as $T \sim 10 \mathrm{mK}, i$. e., the effect is within the experimentally accessible range (see $e$. g., Ref. [4]). To identify the oscillations, one should study the dependence of the period and amplitude of oscillations in $\gamma_{\mathrm{s}}$ on the applied magnetic field $B$. The period increases as $B^{4 / 3}$ and the amplitude grows as $\delta \gamma_{\mathrm{s}}^{\mathrm{osc}} / \gamma_{\mathrm{s}} \propto B^{1 / 3}$ with increasing magnetic field $B$.

In Fig. 1, we schematically present the dependence of amplitudes $A_{k}$ of the $\mathrm{AB}$ oscillations on the energy of Zeeman splitting $g \mu B$ for magnetic impurities, assuming $g \mu B \gg \bar{V}$. If the distance between two magnetic impurities interacting with the strength $g \mu B$ is larger than the mean free path $l$, the amplitude of the $\mathrm{AB}$ conductance oscillations $\mathcal{A}_{k}(B)$ is a monotonic function of the applied magnetic field, described by Eqs. 9a and 111, see region I in Fig. 11 At stronger magnetic fields, defined by Eq. 15, the amplitudes $\mathcal{A}_{k}(B)$ become non-monotonic, see region II in Fig. 11

In summary, we have shown that the amplitude of mesoscopic conductance fluctuations in a dilute magnetic alloy in a high magnetic field is determined by the density of strongly coupled pairs of magnetic impurities. Measurements of the Aharonov-Bohm oscillations in mesoscopic rings may be used as a probe of the distribution function of the interaction strength between magnetic impurities. High-field measurements of the conductance correlation function can provide a direct insight into the oscillatory nature of the RKKY interaction.

The authors are grateful to L. Lévy for turning their attention to the problem and C. Bäuerle and I. Lerner for discussions. V. G. was supported by the US-ONR, LPS, and DARPA; M. V. was supported in part by the MRSEC Program of the National Science Foundation under award DMR 02-13282; L. G. was supported by NSF grants DMR02-37296 and EIA02-10736 (University of Minnesota).

[1] F. Pierre and N. O. Birge, Phys. Rev. Lett. 89, 206804 (2002).

[2] F. Pierre, A. B. Gougam, A. Anthore, H. Pothier, D. Esteve, and N. O. Birge, Phys. Rev. B 68, 085413 (2003).

[3] P. Mohanty and R. A. Webb, Phys. Rev. Lett. 84, 4481 (2000).

[4] F. Schopfer, C. Bäuerle, W. Rabaud, and L. Saminadayar, Phys. Rev. Lett. 90, 056801 (2003).

[5] A. A. Bobkov, V. I. Falko, and D. E. Khmel'nitskii, Zh. Eksp. Teor. Fiz. 98, 703 (1990) [Sov. Phys. JETP 71, 393 (1990)].

[6] M. G. Vavilov and L. I. Glazman, Phys. Rev. B 67, 115310 (2003).

[7] C. Kittel, Quantum Theory of Solids (Wiley \& Sons, New York, 1987).

[8] M. G. Vavilov, L. I. Glazman, and A. I. Larkin, Phys. Rev. B 68, 075119 (2003).

[9] B. L. Al'tshuler, A. G. Aronov, and B. Z. Spivak, Pisma Zh. Exp. Teor. Fiz. 33, 101 (1981) [JETP Lett. 33, 94 (1981)]; D. Yu. Sharvin and Yu. V. Sharvin, Pisma Zh. Exp. Teor. Fiz. 34, 285 (1981) [JETP Lett. 34, 272 (1981)].

[10] A. G. Aronov and Y. V. Sharvin, Rev. Mod. Phys. 59, 755 (1987).

[11] B. L. Altshuler, A. G. Aronov, M. E. Gershenson, and Y. V. Sharvin, Sov. Sci. Rev. Sect. A 9, 223 (1987).

[12] P. G. N. de Vegvar, L. P. Lévy, and T. A. Fulton, Phys. Rev. Lett. 66, 2380 (1991).

[13] A. Larkin, V. Melnikov, and D. Khmelnitskii, Zh. Exp. Teor. Fiz. 60, 846 (1971) [Sov. Phys. JETP 33, 458 (1971)].

[14] A. Larkin and D. Khmelnitskii, Zh. Exp. Teor. Fiz. 58, 1789 (1970) [Sov. Phys. JETP 31, 958 (1970)].

[15] A. Y. Zyuzin and B. Z. Spivak, Pis'ma Zh. Eksp. Teor. Fiz. 43, 185 (1986) [JETP Lett. 43, 234 (1986)].

[16] V. V. Afonin, J. Bergli, Y. M. Galperin, V. L. Gurevich, and V. I. Kozub, Phys. Rev. B 66, 165326 (2002).

[17] S. Hikami, A. I. Larkin, and Y. Nagaoka, Prog. Theor. Phys. 63, 707 (1980).

[18] A. Jagannathan, E. Abrahams, and M. Stephen, Phys. Rev. B 37, 436 (1988).

[19] I. V. Lerner, Phys. Rev. B 48, 9462 (1993). 